\title{
Transient measurement of the anisotropic thermal diffusivity of multilayer carbon fiber reinforced polymers with pulsed thermography
}

\author{
by G. Mayr*, K.-H. Gresslehner*, S. Poechacker* and G. Hendorfer* \\ * University of Applied Sciences, Stelzhamerstrasse 23, 4600 Wels, Austria, guenther.mayr@fh-wels.at
}

\begin{abstract}
The aim of this work is to determine the effective thermal diffusivity tensor for multilayer unidirectional and woven carbon fiber reinforced composites with pulsed thermography. The measurement method utilizes pulsed heating in a geometrical pattern to characterize the thermal material parameters in three orthogonal directions. The dependence of the effective thermal diffusivity on the number of plies is investigated for unidirectional and woven CFRP. In the case of very thin CFRP specimens a strong influence of heat losses, finite pulse duration and heterogeneous material properties on the predicted thermal diffusivity tensor is investigated.
\end{abstract}

\section{Introduction}

The knowledge of the effective thermal diffusivity tensor in three orthogonal directions is important for the numerical simulation of heat transfer problems in complex shaped carbon fiber reinforced plastic (CFRP) components. Ouyang et al. [1] and Sun et al. [2] proposed a measurement technique to determine the anisotropic thermal diffusivity using pulsed heating in a geometrical pattern. The theoretical model used for the prediction of the thermal diffusivity is derived for following ideal conditions: (i) a semi-infinite body in the $x-y$ direction and a finite body in the $z$ - direction, (ii) a dirac-delta heat pulse at $z=0$, (iii) no convection and radiation losses at $z=0$ and $z=L$ (sample thickness) and (iv) a homogeneous material with an anisotropic heat conduction tensor. The aim of this work is to validate the theoretical model for different composite materials (unidirectional and woven fabric) with 2, 4, 8, 16 and 20 layers under experimental conditions.

\section{Experiment}

The experiments were performed with a FPA camera having $320 \times 256$ pixel. Its NETD is about 20 mK at an acquisition rate of 200 frames per second. The temperature was recorded at the back side of the sample $(z=L)$. The thermal excitation was performed at the front side $(z=0)$ with a flash lamp $(6 \mathrm{~kJ}, 2 \mathrm{~ms})$. In figure 1 an image of the local temperature distribution $T(x, y, t)$ is shown. The square in the upper left corner indicates the shielded region at $z=0$. An analytical solution of the heat conduction equation derived by Green's functions method [3] is fitted to the measured temperature profiles for different times. The fit procedure provides the unknown thermal diffusivities in $x, y$ and $z$ direction.

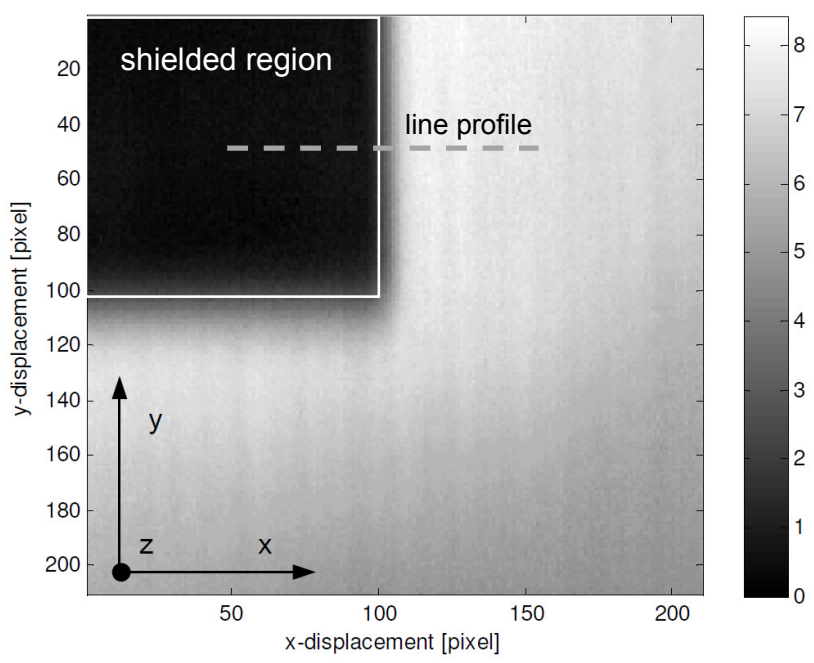

Fig. 1. Image of the local temperature distribution $T(x, y, t)$ at $z=L$ for a unidirectional laminate with 8 plies of CFRP. The square in the upper left corner shows the shielded region at $z=0$. The fibres are aligned in the $y-d i r e c t i o n$. 


\subsection{1/qirt.2016.082}

\section{Results}

A comparison of the experimental data and the theoretical values of a semi - infinite model for four different times after the pulse excitation is shown in figure 2. Especially for short times the predicted temperature values lie closer to the measured values, since there is a negligible influence of the convective heat loss. The dependence of the effective thermal diffusivity on the number of layers is investigated for unidirectional and woven CFRP. In the case of very thin CFRP composites a strong influence of heat losses, finite pulse duration and heterogeneous material properties on the predicted thermal diffusivity tensor is investigated.
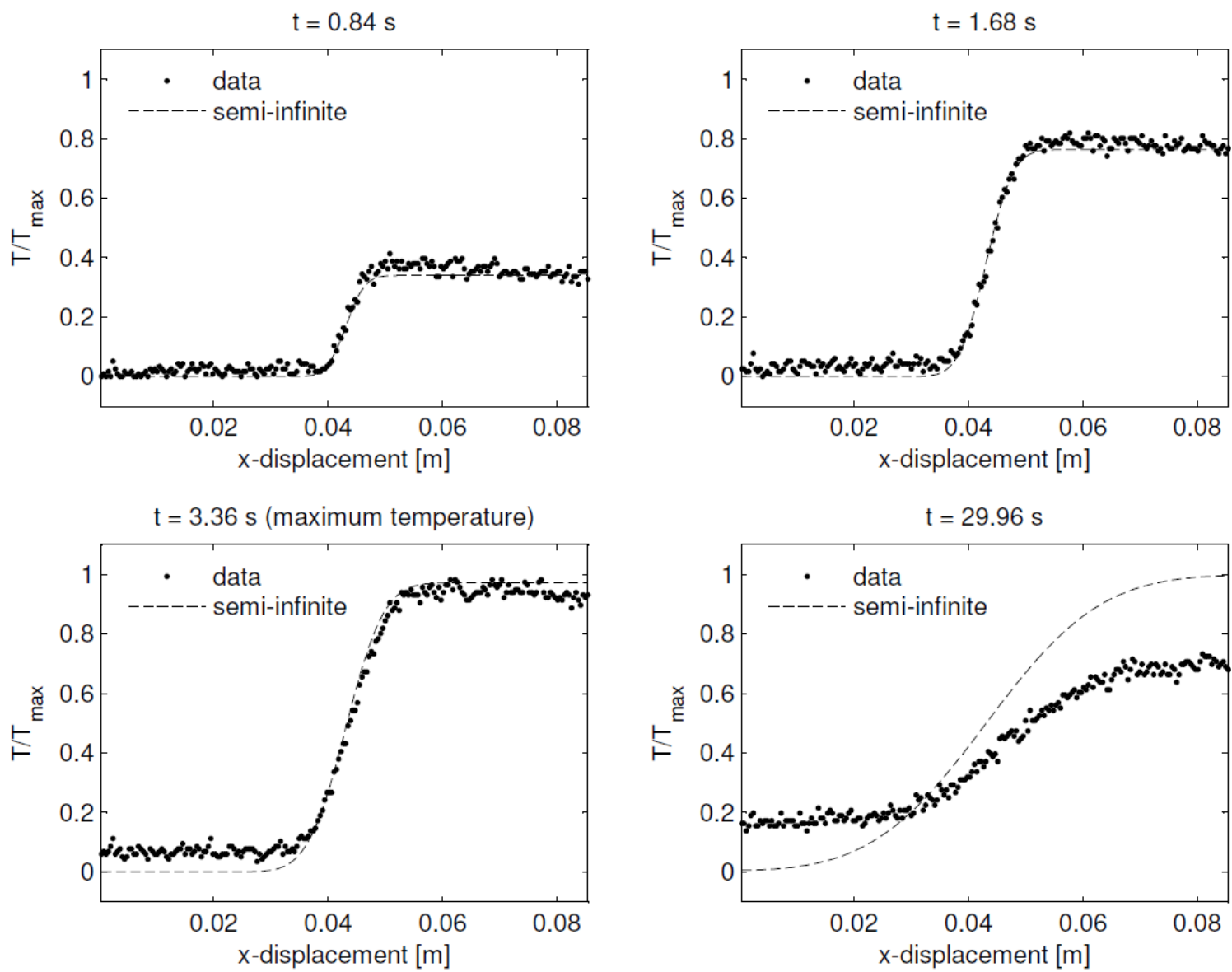

Fig. 2. Normalized temperature profiles at four different times after the thermal excitation. The measured temperature data is fitted to a semi-infinite solution of the heat conduction equation.

\section{REFERENCES}

[1] Ouyang Z., Zhang F., Wang L., Favro L. D., Thomas R. L., Rapid, contactless measurement of thermal diffusivity, Proc. of Review of Progress in QNDE, vol. 17, pp. 453-456 1998.

[2] Sun J. G., Deemer C., Ellingson W. A., "Thermal imaging measurement of lateral thermal diffusivity in continuous fiber ceramic composites", Proc. of 24th Annual Conference on Composites, Advanced Ceramics, Materials, and Structuresnce, vol. 21(3), 2000.

[3] Cole K. D., Beck J. V., Haji-Sheikh A., Litkouhi B. Heat Conduction using Green's Functions. Boca Raton: CRC Press; 2011. 